\title{
Die Schweizer Wenkersätze
}

\author{
Nadja Kakhro (St.Petersburg/Zürich)
}

\begin{abstract}
Georg Wenker, a librarian and a specialist in German Studies of the Marburg University initiated the Linguistic Atlas of the German Language, having started a distribution of a questionnaire composed of sentences with the most striking phonetic and grammar features of the German language. He sent it to elementary teachers who were to translate Wenker's sentences to the dialects they spoke. As a result of responses, dialect borders of the German language were defined - first within Germany, then in the other German-speaking states. In the 1930s those questionnaires, after some changes, got to Switzerland. Having doubts about the method chosen by the researcher and the reliability of the materials received, Wenker's questionnaires were subjected to strong criticism and set aside for a long time. However, Wenker's material is of great interest for researchers, including the Syntax of dialects. Syntactic phenomena are defined in this paper, the study of which became possible due to the questionnaires (by comparison with other sources). Also preliminary results of the analysis of the Swiss questionnaires were presented from the syntactic point of view.
\end{abstract}

\section{$1 \quad$ Aus der Geschichte der Erhebungen}

Georg Wenker, Germanist und Bibliothekar aus Marburg, wurde 1852 in Düsseldorf geboren, studierte in Zürich, Bonn und Marburg und legte in Tübingen 1876 mit der Arbeit "Über die Verschiebung ds Stammsilbenauslautes im Germanischen" das Doktorexamen ab ${ }^{1}$. Schon während der Arbeit an seiner Dissertation entwickelt er Interesse für die räumliche Verbreitung der deutschen Dialekte und die Lautverschiebungsgrenze. 1876 beginnt er dann mit der Umsetzung eines großangelegten Plans: er verfasst einen Fragebogen mit 42 kurzen "volksthümlichen" Sätzen und versendet ihn an die Dorfschullehrer der Rheinprovinz. Es war dabei sein Ziel, die Dialektgrenzen zu ermitteln: "Die hiernach sich ergebende Aufgabe war, in idealer Fassung: die Mundart eines jeden Ortes auf genaueste und vollständigste nach lautlicher, flexivischer, syntactischer und lexicalischer Seite zu erfassen und festzustellen und aus Vergleichung all dieser einzelnen Ortsmundarten erst die Grundsätze, nach denen sie zu Untermundarten und zu Mundarten gruppirt werden müssen, abzuleiten" (Wenker 1881: VI). "Wie aus dem an die Lehrer adressierten Anschreiben hervorgeht, bestand die Aufgabe darin, vorformulierte hochsprachliche Sätze mit den durch das 'allgemein gebräuchliche Alphabet'

\footnotetext{
${ }^{1}$ Im weiteren stütze ich mich auf Ulrich Knoop (1982) und Joachim Herrgen (2001) sowie www.diwa.info.
} 
zur Verfügung gestellten Mitteln in den jeweiligen Ortsdialekt zu übertragen." (www.diwa.info/hist_frageboegen_D.htm).

Das erste Fragebogen-Unternehmen nahm etwa ein Jahr in Anspruch. Aus diesen Erhebungen entstand der "Sprach-Atlas der Rheinprovinz nördlich der Mosel sowie des Kreises Siegen", der erste Sprachatlas weltweit ${ }^{2}$. In den folgenden Jahren wurde allmählich ganz Westfalen, Nord- und Mitteldeutschland erhoben. Aufgrund der während der ersten Auswertungen entstandenen Probleme wurden am ersten, ursprünglichen Fragebogen Änderungen vorgenommen. Insgesamt gibt es neben der rheinischen Version sechs weitere Fassungen des Fragebogens: die westfälische (1877), die erste norddeutsche (1879), die zweite rheinische (1884), die zweite norddeutsche (1887) und die süddeutsche (1887). Die letzte Fassung enthält 40 Sätze.

"Nachdem das Sprachatlasunternehmen 1886 unter staatliche Leitung gestellt worden war, sollte [der Befragungsraum um die süddeutschen Territorien] erweitert werden. Nach Abschluss der Erhebungen lagen insgesamt 44.251 Fragebogen aus 40.736 Schulorten vor." (www.diwa.info/hist_frageboegen_D.htm). Die von 1876 bis 1887 erhobenen Fragebogen wurden in Marburg inventarisiert und regional sortiert. Danach erfolgte die Kartierung. "Die erste fertige Karte lag [bereits] im Jahr 1889 vor. 1923 wurde die [handschriftliche Herstellung von Karten vorerst] eingestellt und mit den Vorarbeiten zu einer Veröffentlichung des Kartenmaterials - dem späteren Deutschen Sprachatlas - begonnen." (www.diwa.info/hist_kartierung_D.htm).

Für die Gebiete außerhalb des Deutschen Reiches wurden eigene Nacherhebungen aufgrund des für den süddeutschen Raum verfassten Fragebogens von 1887 (Mitzka 1952: 14) vorgenommen. "Die erste Nacherhebung wurde 1888 in Luxemburg durchgeführt (325 Bogen), darauf folgten 1926-1933 das Sudetenland (2.854 Bogen), Österreich (3.628 Bogen), Liechtenstein (24 Bogen), das Burgenland (28 Bogen), die Gottschee (35 Bogen), die Schweiz (1.785 Bogen), Polen jenseits der alten Reichsgrenze (396 Bogen), Südtirol (485 Bogen), die sieben und dreizehn Gemeinden der zimbirischen Mundarten in Norditalien (20 Bogen), Nord- und Ostfriesland (67 Bogen). Zusätzlich gingen 2.050 fremdsprachige Bogen ein (z.B. Jiddisch). Damit wurden insgesamt 51.480 Bogen aus 49.363 deutschsprachigen Orten erhoben. Darüber hinaus liegen inzwischen Wenkerbogen aus weiteren deutschsprachigen Gebieten ausländischer Staaten - wie z.B. Russland - vor." (www.diwa.info/hist_frageboegen_D.htm).

\section{Die Fragebogen-Aktion in der Schweiz}

Mein momentanes Interesse gilt den Nacherhebungen, die in der Schweiz durchgeführt worden sind. Vom Phonogrammarchiv der Universität Zürich ging schon Anfang des 20. Jahrhunderts die Initiative aus, Tonaufnahmen der Wenkersätze zu machen, die bekanntlich nicht zur Tonaufnahme vorgesehen waren (cf. Fleischer/Gadmer 2002: 47-50). Sie wurden in Zürich als Vergleichsmaterial aufgezeichnet. Man weiss nicht genau, welche Fassung der

\footnotetext{
2 Das Vorhaben stand aber zweifellos in der Tradition europäischer Dialektforschung (cf. Knoop 1982: 38-46).
} 
Wenkersätze für die entsprechende Aufnahmeserie Verwendung fand. Wahrscheinlich orientierte man sich für die Zürcher Aufnahmen an der Fassung, die auch Joseph Seemüller, Leiter des Phonogrammarchivs zu Wien, verwendete und in den Deutschen Mundarten abdruckte. Seemüller hat aber die rheinische Syntax der hochdeutschen Vorlage nicht übernommen und den Wenkerschen Satz dass die Milch bald an zu kochen fängt in dass die Milch bald zu kochen anfängt umformuliert (cf. Fleischer/Gadmer 2002: 47-50). Aber bereits nach 1910 wurden die Wenkersätze vom Zürcher Phonogrammarchiv nicht weiter beachtet. Das scheint darauf hinzudeuten, dass die damit gemachten Erfahrungen nicht befriedigend waren. Erst 2002 gewannen diese Aufnahmen die Aufmerksamkeit der Forscher und wurden auf digitale Träger übertragen und veröffentlicht.

$\mathrm{Zu}$ der schriftlichen Erhebung kam es erst später und auf Initiative Marburgs hin. Im Vorwort zur 6. Lieferung des Deutschen Sprachatlas von 1932 schreibt Ferdinand Wrede (1932: Vorwort), Wenkers Nachfolger: "Der Anschluss der deutschen Schweiz ist jetzt gesichert dank den weiteren Verhandlungen, die Prof. Dr. Jacobsohn, z.Z. Direktor unserer Zentralstelle hier in Marburg, sowohl mit der schweizerischen Gesandschaft in Berlin wie mit Prof. Dr. Bachmann in Zürich geführt hat. Wir hoffen übers Jahr mehr darüber berichten zu können." Die ersten offiziellen Verhandlungen über die Durchführung der Bogen-Aktion in der deutschsprachigen Schweiz begannen auf Staatsniveau offensichtlich mit der Note der Deutschen Gesandtschaft an das Eidgenössische Politische Departement vom 30. Oktober 1931 (Briefe vom 06.05.1932 und 18.11.1932), in der gefragt wurde, ob die schweizerischen Behörden bereit wären, dabei mitzuwirken. In seiner Antwort vom 18. November 1932 erklärte sich das Eidgenössische Departement des Innern bereit, die Fragebogen an die Erziehungsdirektionen der Kantone zu verteilen, wonach diese die Fragebogen mit einer Empfehlung an die Lehrer ihrer sämtlichen Primarschulen übermitteln und nachher wieder einsammeln würden. Den ersten Schätzungen nach benötigte die Schweiz insgesamt ca. 2900 Formulare der Fragebogen, um diese folgendermassen zu verteilen:

Zürich - 349 Bogen, Bern - 663, Luzern - 250, Uri - 26, Schwyz - 70, Obwalden - 20, Nidwalden - 20, Glarus - 50, Zug - 21, Freiburg - 52, Solothurn - 150, Basel-Stadt - 12, Baselland - 72, Schaffhausen - 50, Appenzell A.-Rh. - 50, Appenzell I.-Rh. - 2, St.Gallen 250, Graubünden - 120, Aargau - 250, Thurgau - 180, Wallis - 92 (Schreiben vom 14. November 1932 und vom 18. November 1932).

Aus der Korrespondenz des Sprachatlasses in Marburg und des Sprachatlasses in Zürich geht hervor, dass im Jahr 1933 die Erhebungen schon "in Fluss gekommen" (Schreiben vom 18. März 1933) waren, obwohl sich die schweizerische Direktion des Unterrichtswesens "keine richtige Vorstellung von Umfang und Dauer der ihr [...] zugedachten Arbeit machen" konnte (Schreiben vom 18. November 1932). Bereits Ende des folgenden Jahres vermerkt Walter Mitzka (1934: Vorwort) im Vorwort zur 7. Lieferung des Deutschen Sprachatlasses: "Inzwischen haben wir aus der Schweiz 1785 Antworten auf den Wenkerschen Fragebogen durch den verstorbenen Prof. Albert Bachmann, der unser Unternehmen stets hochherzig gefördert hat, erhalten. Ihm und der Mittelstelle des Schweizerischen Idiotikons sagen wir im 
Namen der Sprachwissenschaft herzlich Dank. Die Ergebnisse der Antworten bringen wir von der 8. Lieferung an."

Die Schweizer Wenkerbogen wurden im Archiv des Sprachatlasses in Marburg aufbewahrt, Kopien - im Archiv des Schweizerdeutschen Sprachatlasses in Zürich. Da die schweizerischen Fragebogen als solche offenbar nie inventarisiert wurden, kann man nur auf Grund der indirekten Daten und des Bestandes der erhalten gebliebenen Bogen vermuten, wie viele es genau waren. Mit der Zeit hat sich die Anzahl der Schweizer Bogen möglicherweise etwas reduziert, was wahrscheinlich auf die Umzüge des Marburger Archivs, eventuelle Verluste und Mangel an Interesse zurückzuführen ist. Laut Mitzka (1934: Vorwort) waren es 1785, im Deutschen Seminar der Universität Zürich sind bislang nur 1768 Kopien ermittelt worden ${ }^{3}$.

Bereits zu Wenkers Zeiten gab es vielfach Kritik an seiner schriftlichen Methode (cf. Haas 1995), weshalb die Daten in der Schweiz zwar erhoben wurden, aber unausgewertet blieben. Die Kritik am Wenkerschen Werk betraf vor allem die Unvollkommenheit der Transkriptionssysteme und die Tatsache, dass er nur einen Bogen pro Ort als repräsentativ ansetzte (cf. Bremer 1895) und dass auf Grund der fehlenden Informantenkontrolle im Sprachatlas noch nicht einmal erkennbar ist, welcher sozialen Schicht erhobene Dialektvarianten jeweils zuzuordnen seien (cf. www.diwa.info). Ausserdem wurde dem Forscher vorgeworfen, dass seine Sätze künstlich formuliert seien und in der spontanen Rede eines nativen Sprechers kaum entstehen können. Man liest bei Arno Ruoff (1965: 102): "Auch ein begabter Sprecher ist einfach nicht fähig, die Satzvorlagen in seine Mundart zu transponieren, vor allem wenn es in dieser jene Sätze gar nicht geben kann." Er betont auch, dass jede auf den Wenkerbogen beruhende Sprachanalyse kaum möglich ist, denn "wenn dem Sprecher die Übersetzung im lautlichen oder sogar im lexikalischen Bereich doch gelingt, dann doch gewiß nicht mehr im syntaktischen oder stilistischen: da kann er es nicht und wagt es auch nicht, weil er oft viel zu weit von der Vorlage abweichen müßte" (cf. 1965: 102). Andere Sprachwissenschaftler waren hinsichtlich der Wenkersätze auch dieser Meinung (cf. Schirmunski 1959: 83-87) und haben die Brauchbarkeit des gesammelten Korpus in Frage gestellt. In diesem Zusammenhang ging das Interesse an den Wenkerschen Materialien verloren, und die Bogen gerieten für eine Zeit lang in Vergessenheit.

Auch die Syntax als eines der Forschungsgebiete der Sprachwissenschaft genoss bis auf die letzten Jahre wenig Aufmerksamkeit der Dialektologen. Iwar Werlen (1994: 49) schreibt: "ein Blick in Bibliographien zur deutschen Dialektforschung zeigt: das Hauptinteresse der Forscher und Forscherinnen lag und liegt nicht auf dem Gebiet der Syntax." Ernst Schwarz (1950: 118) nennt die Syntax "das Stiefkind der Dialektologie". Viele Wissenschaftler und Wissenschaftlerinnen zweifelten und zweifeln am Vorhandensein und an der Raumbildung der Dialektsyntax, indem sie diese als Syntax gesprochener Sprache betrachten und Dialekte für regellos halten. Man findet bei Löffler (2003: 109): "[...]Die Dialektologie arbeitet letzten Endes immer komparativ. Für historische und geografische Vergleiche bietet die Syntax

\footnotetext{
3 Ohne Liechtenstein. Das sind Kopien der in Marburg und Zürich aufbewahrten Bogen, die für das von Prof. Glaser geleitete Dialektsyntax-Projekt gemacht wurden.
} 
wenig Anhaltspunkte. [...] Der Hauptgrund, weshalb mundartliche Syntax wenig bearbeitet ist, liegt jedoch in der nicht unbegründeten Annahme, dass dialektale Syntax sich von hochsprachlicher kaum unterscheidet"; und etwas vorsichtiger: "Die vorläufigen Ergebnisse zeigen, dass, die syntaxgeprägten Räume sehr großflächig sind und dass es nur sehr wenige syntaktische Variablen sind, die raumbildend wirken" (cf. 2003: 116). Erstaunlich ist, dass angesichts dieser Forschungslücke die Wenkersätze, die zwar aus lautlichem Interesse heraus abgefragt wurden, aber dennoch Sätze darstellen, nie systematisch untersucht wurden ${ }^{4}$.

Bezüglich der Wenkersätze und ihres syntaktischen Wertes findet man allerdings in der "Deutschen Mundartkunde" von V.M. Schirmunski (1956: 379) folgende Einschätzung: "[...]Obwohl es einige Arbeiten zur Syntax gibt, die zum Teil allgemein wissenschaftlich, zum Teil fachspezifisch ausgerichtet sind, und die Syntax eines Dialektes bzw. seine einzelnen syntaktischen Besonderheiten beschreiben, fehlt es an dialektgeographischen Forschungen, die syntaktische Phänomene in ihrer lokalen Verteilung betrachten. Die Wenker-Bogen enthalten diesbezüglich keinerlei vertrauenswürdiges Material, denn die Methode, die in der Hochsprache vorgegebenen Sätze in den Dialekt zu übersetzen, führt unvermeidlich zu syntaktischen Kalkierungen, die die Formen der lebendigen volkstümlichen Umgangssprache verdrehen." Eine syntaktische Auswertung der Wenkersätze muss also besonders begründet werden.

\section{$3 \quad$ Erste Ergebnisse der Auswertung}

Es ist zu bemerken, dass nachdem die Syntax einen Aufschwung in der Grammatik überhaupt erlebt hat, findet sie in jüngster Zeit vermehrt in der Dialektologie Berücksichtigung, was die zahlreichen laufenden Projekte zur Forschung der Dialektsyntax, die an schweizerischen sowie deutschen Universitäten durchgeführt werden, anschaulich machen (cf. Glaser 2000).

Parallel zu einem unter der Leitung von Elvira Glaser an der Universität Zürich durchgeführten Projekt zur schweizerdeutschen Syntax ist die Idee entstanden, auch das reiche Wenkersche Material syntaktisch auszuwerten und auf diese Weise syntaktische Erscheinungen aus der historischen Perspektive zu betrachten bzw. die Ergiebigkeit des Wenkermaterials zu testen. Die Sätze haben sich mittlerweile als nicht ununteressant in syntaktischer Hinsicht erwiesen. Der syntaktischen Auswertung des in der Schweiz in den 30er Jahren erhobenen Materials ist mein Dissertationsprojekt gewidmet. Die Arbeit ist noch nicht abgeschlossen, jedoch hat die Auswertung (ca. 1200 von 1768 Bogen $^{5}$ ) einige Ergebnisse gebracht, die hier vorgestellt werden.

Die in der Schweiz eingesetzten Bogen enthalten 40 Sätze (cf. Anhang), die ihrem Bau nach vorwiegend einfache Aussagesätze sind, zweigliedrig und mit der Erststellung des Subjekts, dazu finden sich auch Fragesätze und ein Aufforderungssatz. Ausserdem gibt es einige Parataxen alle aus zwei Elementarsätzen bestehend und grösstenteils asyndetisch sowie zwei konjunktional verbundene Sätze. Hypotaxen sind weniger vertreten. Unter den Nebensätzen

\footnotetext{
${ }^{4}$ Ausnahme bildet der Satz 24. (Cf. Maurer 1924).

5 Teilweise ausgewertet sind Bogen aus den Kantonen Aargau, Bern und Luzern. In diesen Kantonen sind bis jetzt nur die Infinitiv-Anschlüsse ausgewertet. Alle anderen Kantone sind vollständig ausgewertet worden.
} 
findet sich ein Temporalsatz, einige Finalsätze und nicht eingeleitete Objektsätze mit der indirekten Rede. Nach der teilweisen Auswertung der Bogen hat sich ergeben, dass die Sätze tatsächlich eine Reihe von syntaktischen Phänomenen aufweisen, die regionale Verteilung zeigen. Unter solchen Phänomenen, die vom sprachgeographischen Aspekt aus einer Untersuchung wert sind, sind zu nennen (alle Beispiele sind in der hochdeutschen Schreibung angegeben):

Artikelgebrauch mit Stoffnamen (Satz 19: Wer hat mir meinen Korb mit Fleisch/mit dem Fleisch gestohlen? etc.)

Ausfall des pronominalen Subjekts (Satz 18: Hättest du ihn gekannt!/Hättest ihn gekannt!) Flektierter Prädikativ (Satz 15: Du hast heute am meisten gelernt und bist artig/artigs gewesen...; Du bist noch nicht gross genug/nicht grosses genug...)

Präpositionale Dativ-Markierung (Satz 21: Wem/A wem hat er die neue Geschichte erzählt?)

Präpositional-adverbialer Rahmen (Satz 3: Tu Kohlen in den Ofen/in den Ofen ine; Sein Bruder will sich zwei schöne neue Häuser in eurem Garten/in eurem Garten ine bauen)

Infinitiv-Anschlüsse (Satz 2, Satz 16: Es hört gleich auf (zu)/mit schneien...; Du bist noch nicht groß genug, um eine Flasche Wein auszutrinken/zum eine Flasche Wein auszutrinken etc.)

Reihenfolge pronominaler Satzglieder (Satz 18: Hättest du ihn/ihn du gekannt; ...und habe es ihr/ihr es gesagt)

Stellung der Modalverben in verbalen Komplexen (Satz 37: [...]sie haben verkaufen wollen/wollen verkaufen)

Tun-Periphrase (Satz 2: Es tut gerade aufhören schneien [...]etc.)

Verbverdoppelung (anfangen, gehen) (Satz 3: Tue Chole in Ofu, dass d'Milch bald faht afe choche; Satz 17: Gang, bis so guet u. dire Schwöschter go säge, si soll d'Chleider fur eueri Muetter fertig nahe u. mit dr Bürschte suuber mache).

Im folgenden will ich auf einige Beispiele näher eingehen, um die Variabilität der Ausdrucksmittel aufzuzeigen. Unter diesem Aspekt ist das Problem des Infinitiv-Anschlusses besonders interessant, denn er bietet eine breite Palette der Ausdrucksmöglichkeiten einerseits und regionale Variation andererseits. Als Erstes kann in den Wenkersätzen der Gebrauch des Infinitivs mit Phasenverben beobachtet werden: mit Verben des Beginnens (Satz 3) und des Aufhörens (Satz 2). Die Verben des Beginnens sind in den Schweizer Bogen mit dem Verb afange "anfangen" vertreten. Als Verben des Aufhörens treten neben dem Verb ufhöre "aufhören" mehrere Synonyme auf: höre "hören", na loh "nachlassen", na gebe "nachgeben". Der Infinitiv wird bei diesen Verben meistens ohne die Partikel "zu" gebraucht: mit den Verben des Beginnens in 68\% der analysierten Bogen, mit den Verben des Aufhörens - in 78\%. Der Infinitiv mit der Partikel "zu" wird mit den Verben des Beginnens in 5,5\% der Fälle gebraucht, mit den Verben des Aufhörens - in 15\%: Es ist zu vermerken, dass im Kanton Appenzell-Innerhoden neben "zu" die Partikel "ane" verwendet wird. Mit den Verben des Aufhörens wird in einigen Ortschaften diese Konstruktion durch eine alternative Konstruktion mit dem substantivierten Infinitiv und der Präposition "mit" ersetzt. Im Vergleich zu der "zu"-Konstruktion kommt diese nur in 5,5\% der Bogen vor. 
Im Satz 16 kommen verschiedene Variationen der Infinitiv-Anschlüsse vor. Als häufigste finale Konstruktion ist für $+\left(z^{\prime}\right)+$ Infinitiv zu bezeichnen, sie kommt in 39\% der Bogen vor, als nächstes folgt $u m+\left(z^{\prime}\right)+$ Infinitiv $(30 \%)$, zum $+(z)+$ Infinitiv $(23 \%),\left(z^{\prime}\right)+$ Infinitiv (5\%). Im Gegensatz zu den Infinitiv-Anschlüssen mit Phasenverben werden diese finalen Konstruktionen vorwiegend mit der Partikel "zu" verwendet. Für die verschiedenen Varietäten ergeben sich z.T. areale Verteilungen. Das sieht man auf den Karten 1-4.

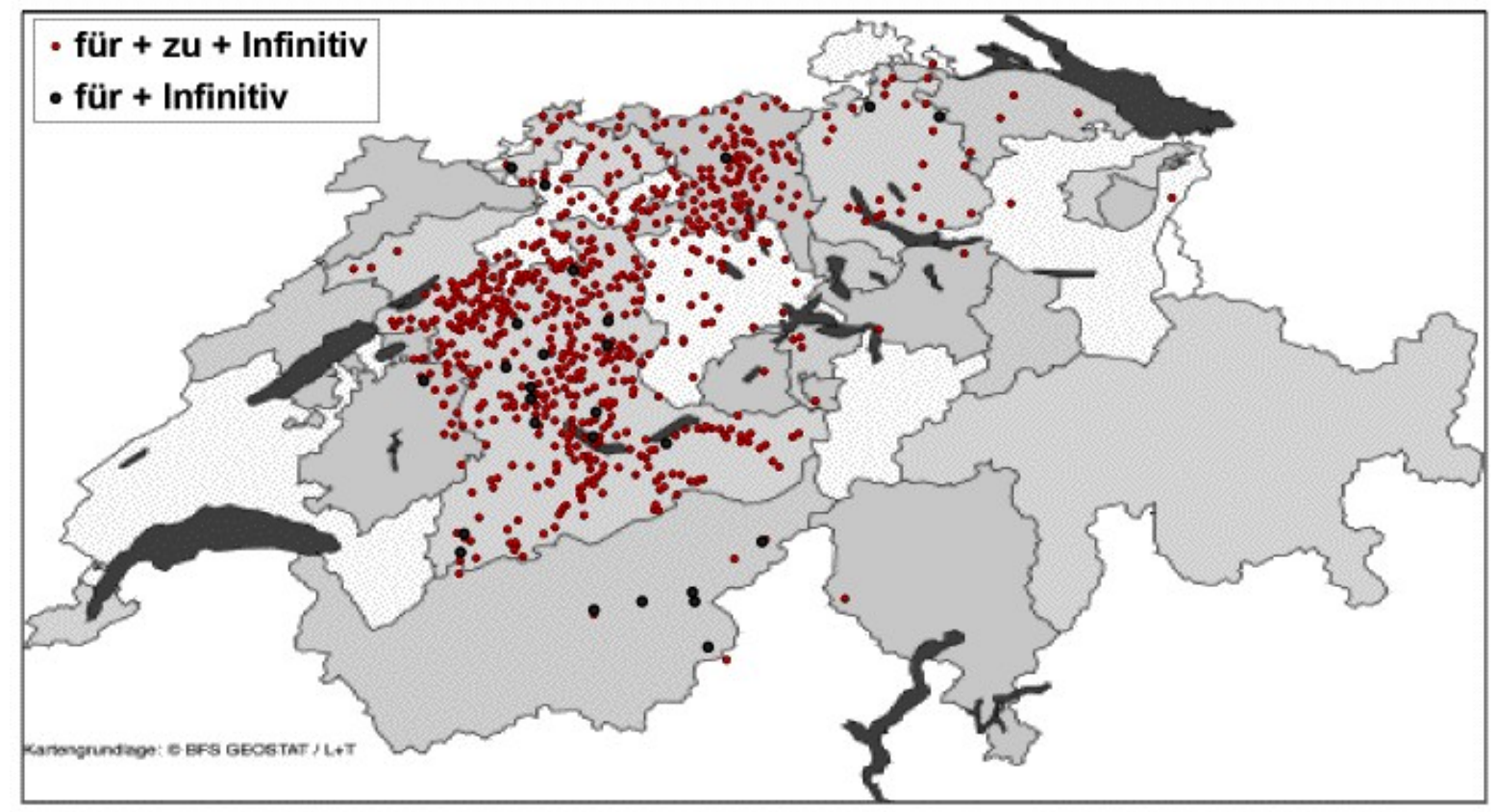

Karte 1

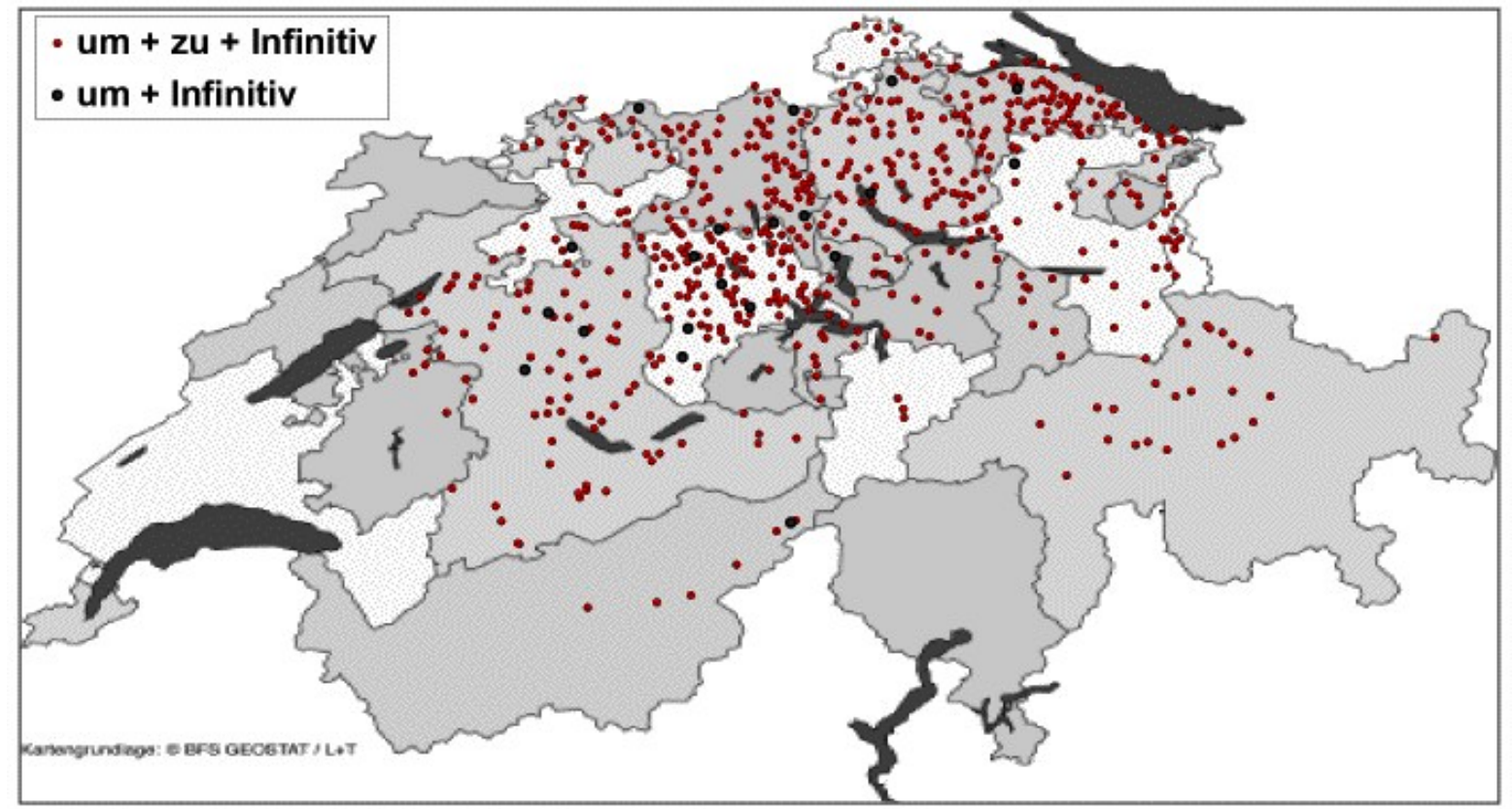

Karte 2

\footnotetext{
${ }^{6}$ Das häufige Vorkommen der "um...zu"-Konstruktion könnte auf hochdeutschen Einfluss zurückzuführen sein, der bei der schriftlichen Übersetzung unvermeidlich ist.
} 


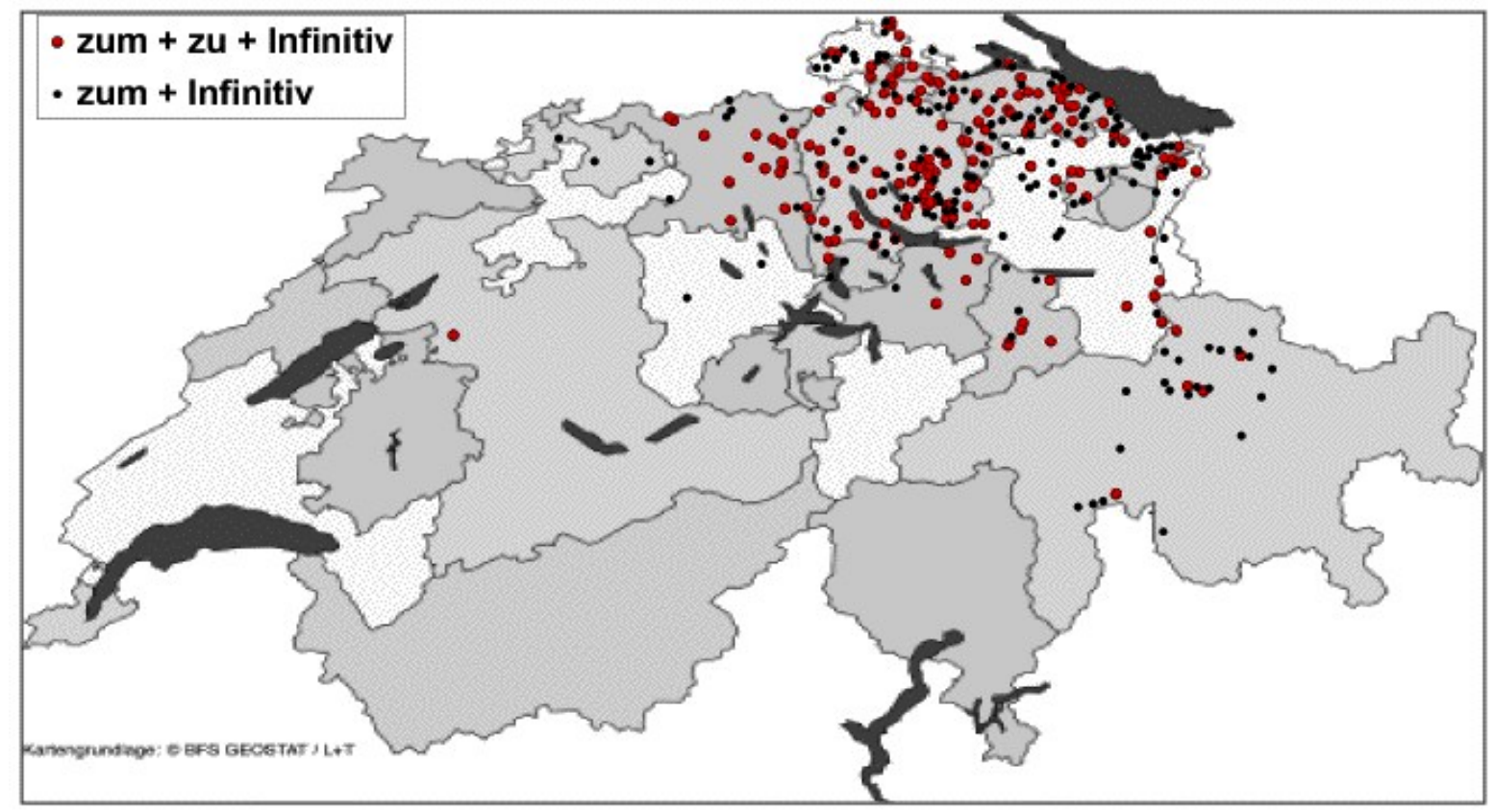

Karte 3

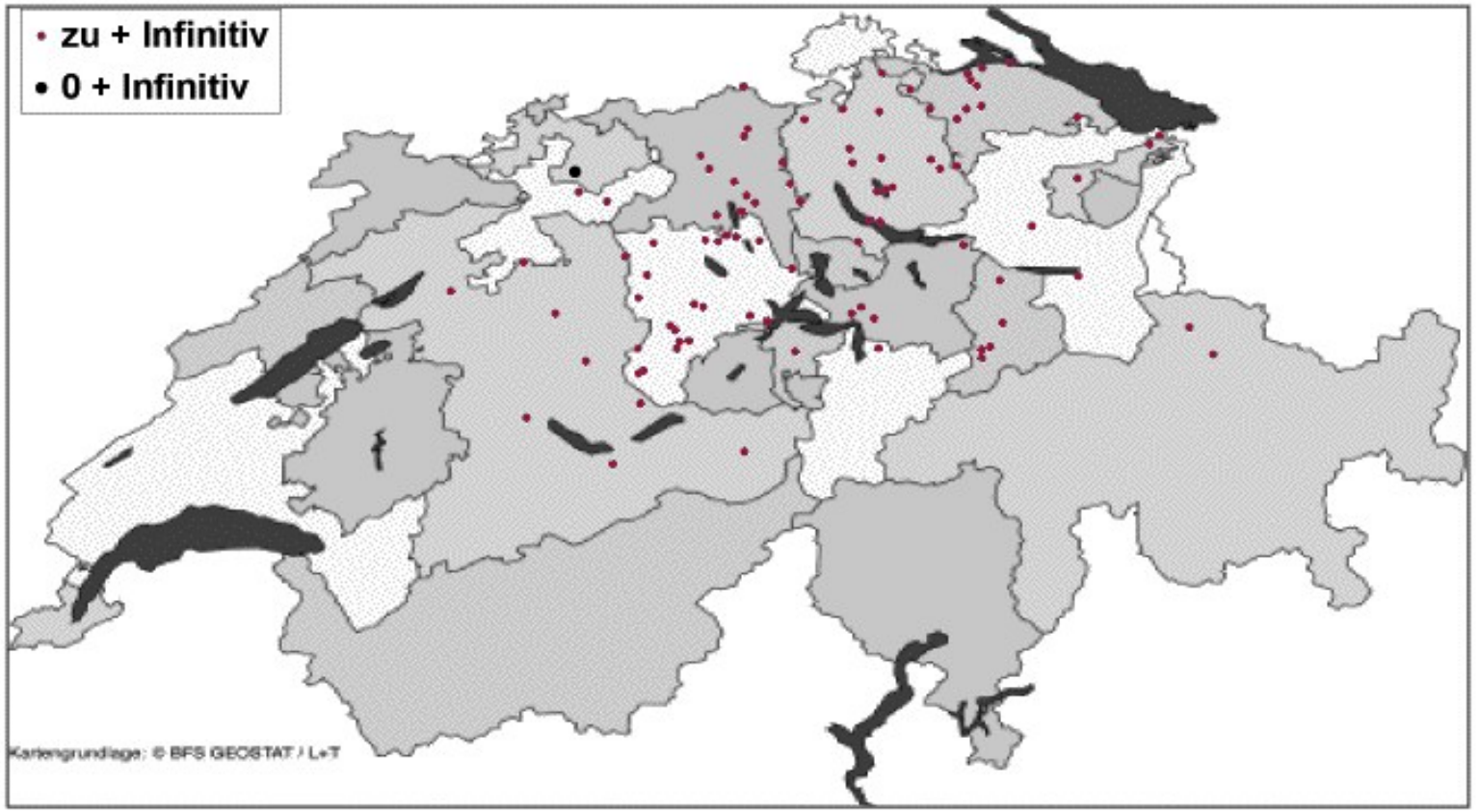

Karte 4

Die Konstruktion für $+\left(z^{\prime}\right)+$ Infinitiv ist eher für den Westen typisch, die Konstruktion zum $+\left(z^{\prime}\right)+$ Infinitiv kommt öfter im Osten vor (cf. Glaser 2003, Seiler (im Dr.)).

Aus den Karten geht auch hervor, dass der Infinitiv in diesen finalen Konstruktionen vorwiegend mit Hilfe der Partikel "zu" angeschlossen wird, z.B. für $+z^{\prime}+$ Infinitiv wird häufiger verwendet als für + Infinitiv etc. Eine Ausnahme bildet in diesem Falle die Konstruktion zum $+\left(z^{\prime}\right)+$ Infinitiv, denn die Variante ohne die Partikel "zu" öfter vorkommt als die Variante mit "zu". Ausserdem weist diese Konstruktion, die vor allem in den Ostschweizer Dialekten vorkommt, noch eine Variante auf, und zwar zunere $+\left(z^{\prime}\right)+$ Infinitiv, 
wo an die Partikel der unbestimmte Artikel weiblichen Geschlechts angehängt wird (Diesbach GL; Eschenz TG; Vorderthal SZ).

In den Sätzen, wo explizit oder implizit eine Bewegung zum Ausdruck kommt, kann der Infinitiv von "go" begleitet werden. Diese Erscheinung kommt in den Sätzen 9, 17, 24, 25, 38 vor (z.B. Bis so guet u. gang diner Schwöschter go säge, si soll d'Chleider fur eueri Muetter fertig nähe u. mit dr Bürschte suuber bürschte). Die regionale Verteilung dieser Erscheinung lässt sich vorläufig auf die Ostschweiz und punktuelles Vorkommen in der Zentralschweiz beschränken (Karte 5)

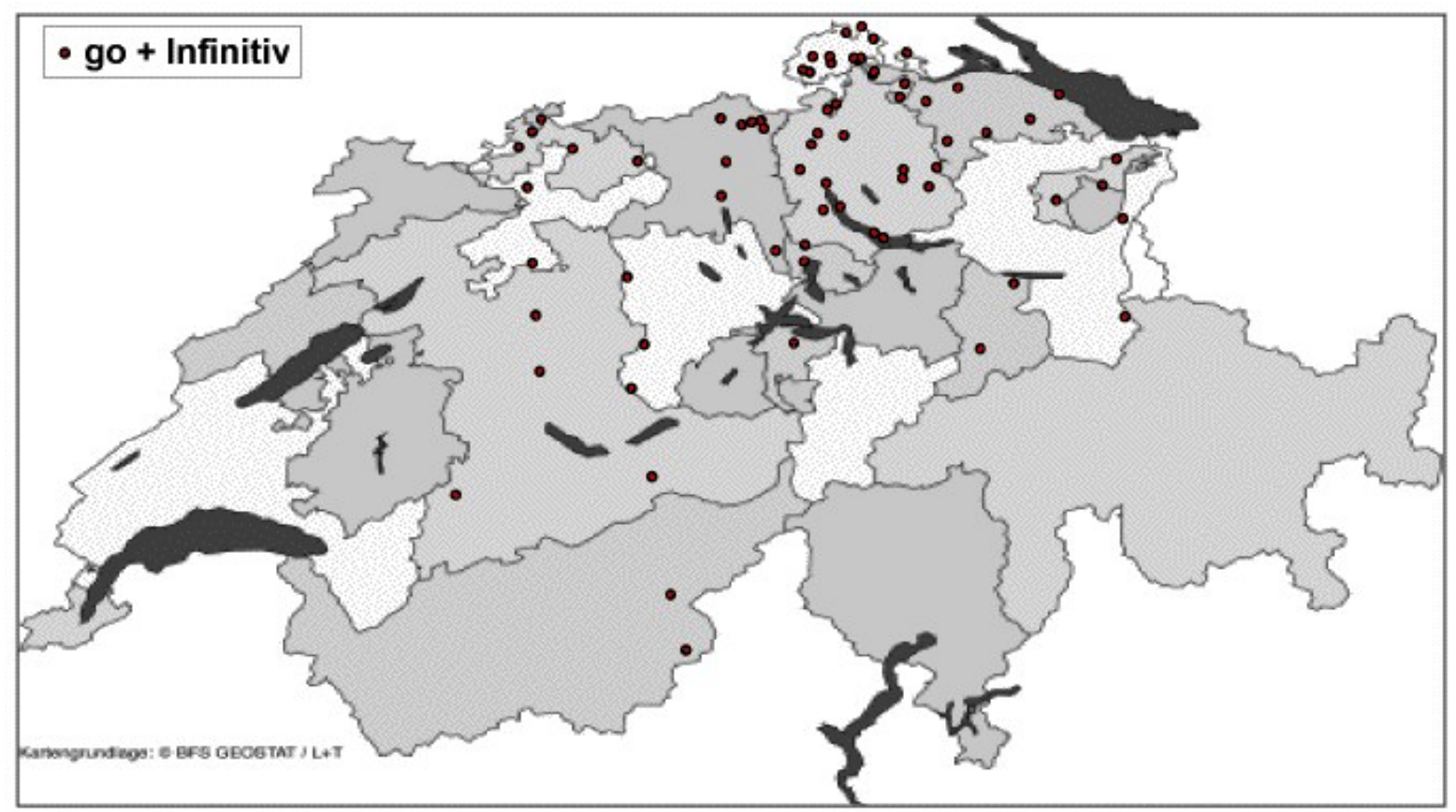

Karte $5^{7}$

Als nächstes Phänomen, das in den Wenkersätzen untersucht werden kann, ist die Wortfolge im Satz zu nennen. Dabei handelt es sich nicht nur um die Stellung der Hauptglieder, des finiten Verbs bzw. des Modalverbes, was zweifellos zu interessanten Ergebnissen führen kann (cf. Maurer 1926, König 1978), sondern auch um die Abfolge der nominalen und pronominalen Satzglieder. Im Satz 18 "Hättest du ihn gekannt..." tauschen das AkkusativObjekt und das Subjekt ihren Platz. Das wird aus den Karten 6 und 7 ersichtlich:

\footnotetext{
${ }^{7}$ Diese Karte und die weiteren Karten sind vorläufige Karten, auf denen noch ein Teil der Bogen aus den Kantonen Aargau, Bern und Luzern fehlen.
} 


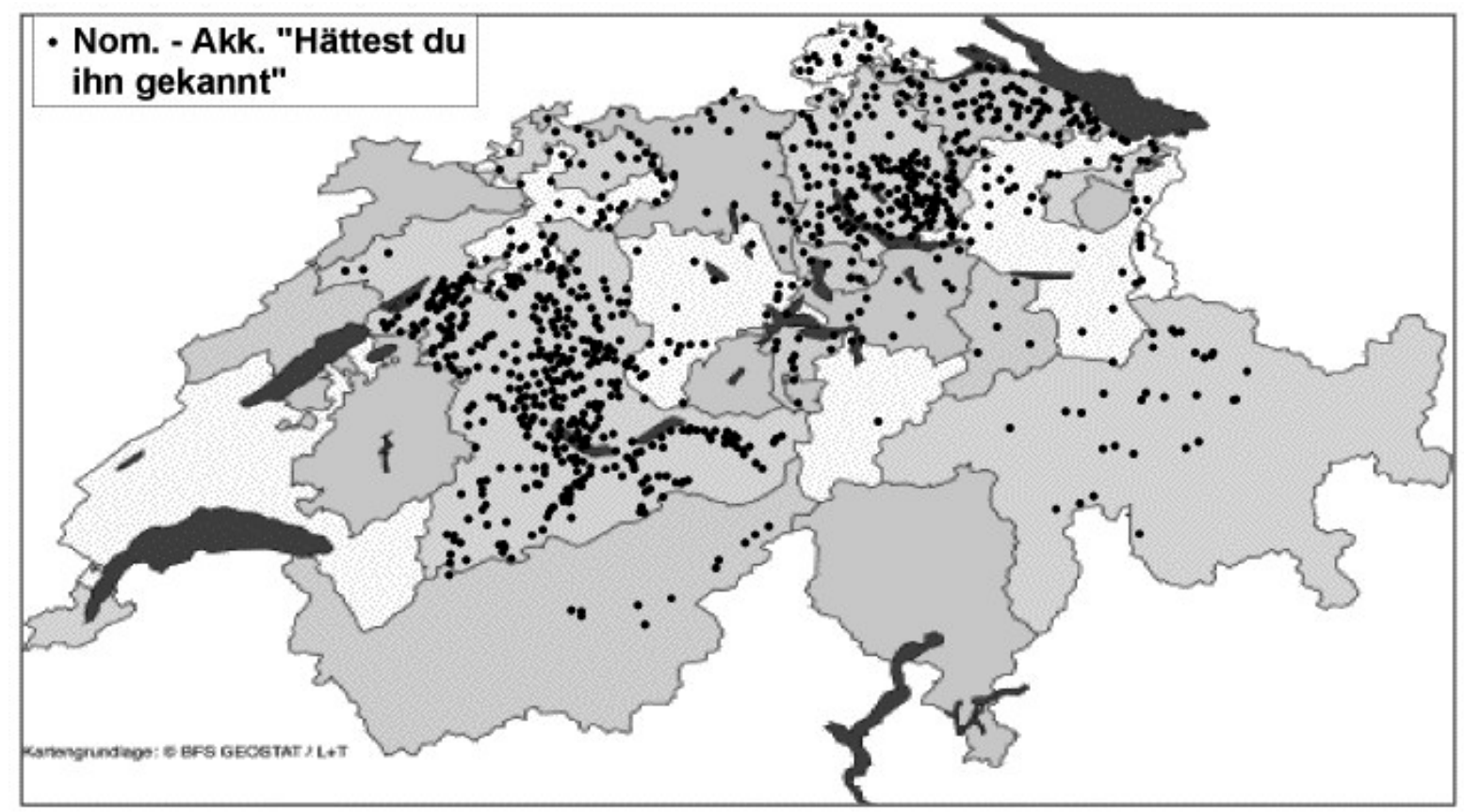

Karte 6

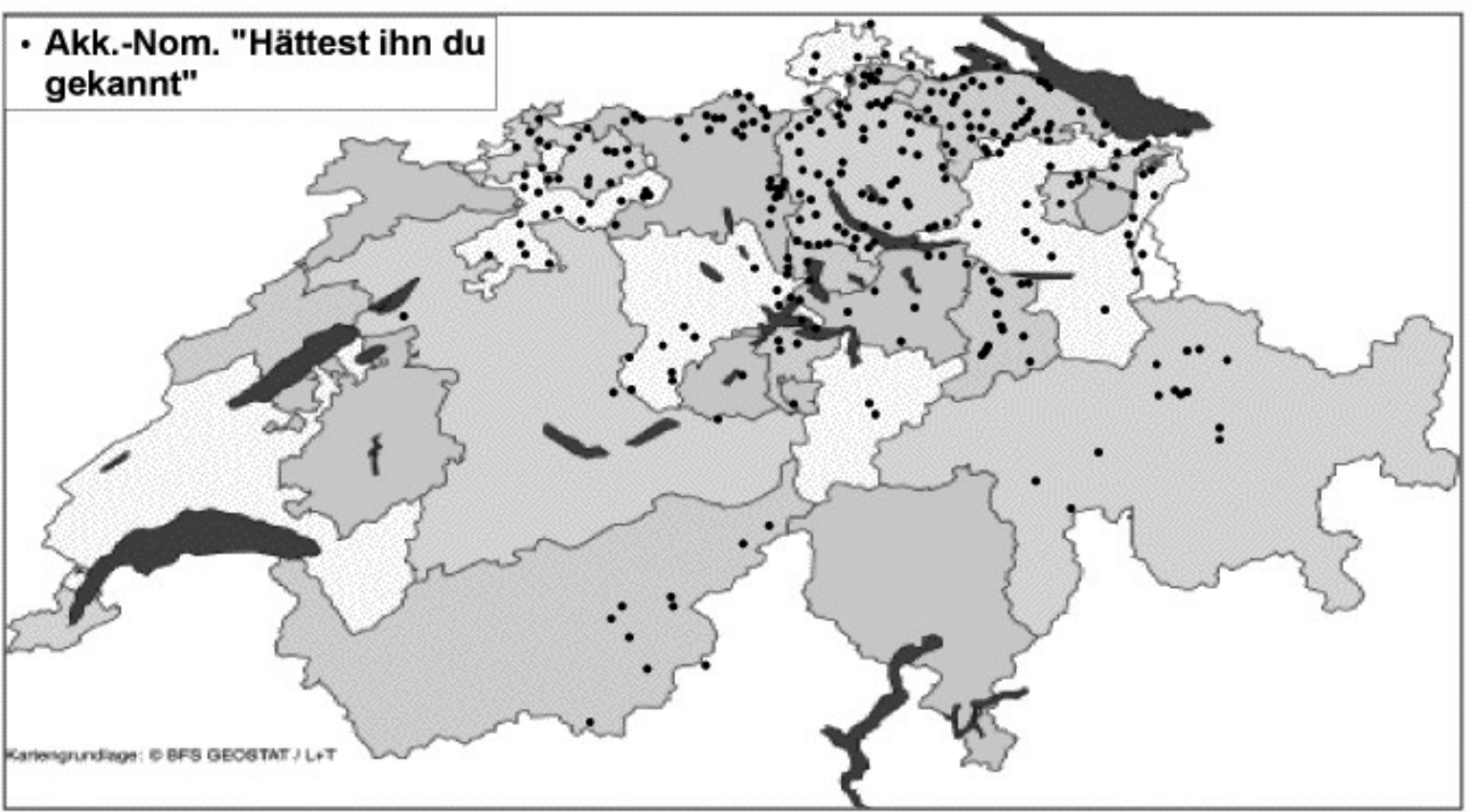

Karte 7

Die Abfolge $i h n d u$ ist vor allem in der Ost- und Zentralschweiz zu beobachten, während $d u$ ihn keine areale Verteilung zeigt.

Ein ähnliches Phänomen kommt im Satz 9 vor "Ich bin bei der Frau gewesen...". Im Gegensatz zur schriftdeutschen Abfolge des Dativ- und Akkusativ-Objekts im Hauptsatz (cf. Duden 1998: 822 ff.) geht dem Akkusativ-Objekt das Dativ-Objekt voran, was man auf den Karten 8 und 9 sehen kann. Die Reihenfolge Akkusativ - Dativ ist eher für den Osten typisch als für den Westen, und die hochdeutsche Reihenfolge ist überall verbreitet. 


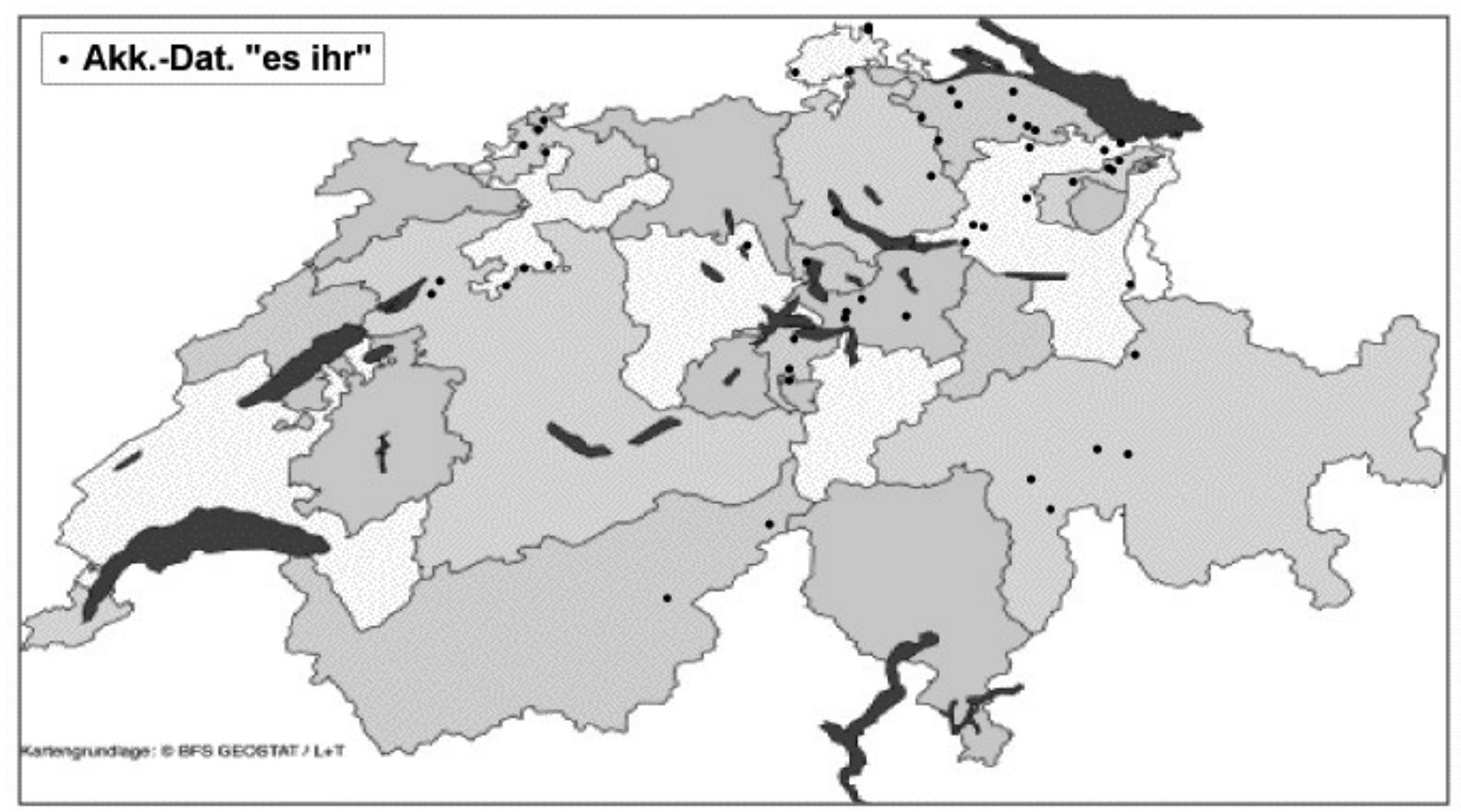

Karte 8

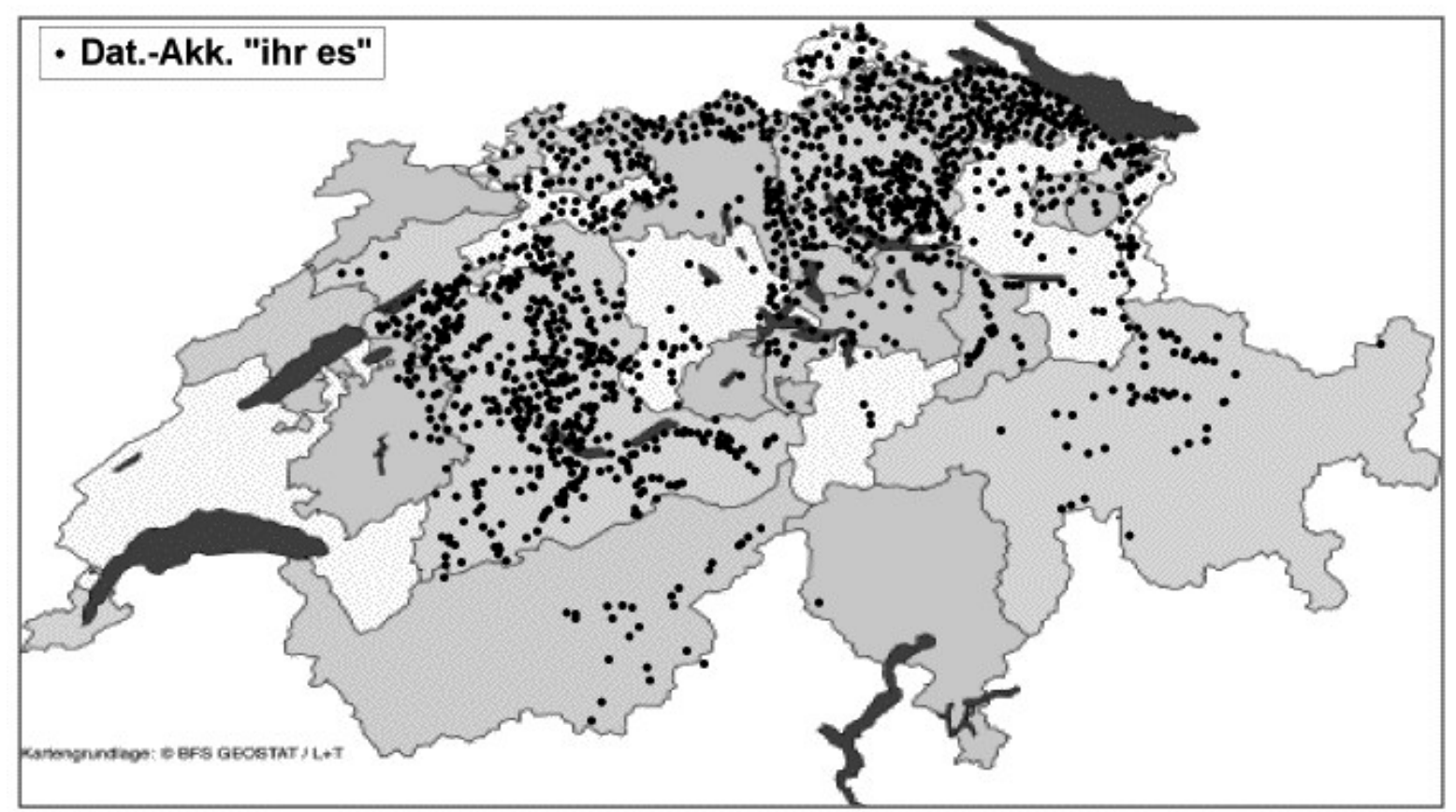

Karte 9

Die syntaktische Auswertung der Schweizer Wenkersätze ist momentan im vollen Gange. Wenn man die ersten hier aufgeführten Ergebnisse mit den Daten der modernsten syntaktischen Dialektuntersuchungen vergleicht, sieht man, dass die Schweizer Wenkerbogen ein klares dialektgeprägtes Bild ergeben und nicht, wie es behauptet wurde, die standarddeutsche Vorlage wiederholen. Es sei betont, dass die Informanten, wie es aus den ausgewerteten Bogen hervorgeht, sehr ernsthaft die gestellte Aufgabe erfüllt und die in der hochdeutschen Vorlage stehenden für den Dialekt unmöglichen Konstruktionen vermieden 
haben, indem sie eigene, zum Teil umformulierte und dennoch für die sprachliche Analyse interessanten Varianten gewählt haben.

Trotz der jahrelangen Kritik am Wenkerschen Werk erweist sich das Anfang des 20.Jahrhunderts gesammelte Material als eine gerade auch vom syntaktischen Standpunkt aus wertvolle Datenquelle über eine der Entwicklungsphasen der Dialekte.

\section{Literaturangaben}

Bremer, Otto (1895): Beiträge zur Geographie der deutschen Mundarten in Form einer Kritik von Wenkers Sprachatlas des deutschen Reichs. Leipzig.

Duden (1998): Die Grammatik der deutschen Gegenwartssprache. 6. Auflage. Dudenband 4. Mannheim, Leipzig, Wien etc.

Fleischer, Jürg/Gadmer, Thomas (eds.) (2002): Schweizer Aufnahmen (deutsch). (Sound Documents from the Phonogrammarchiv of the Austrian Academy of Science. The Complete Historical Collections 1899-1950 6/1, 6/2) Wien, Zürich

Glaser, Elvira (2000): "Erhebungsmethoden dialektaler Syntax". In: Stellmacher, Dieter (ed.): Dialektologie zwischen Tradition und Neuansätzen. Stuttgart (= ZDL-Beiheft 109): 258277.

Glaser, Elvira (2003): "Schweizerdeutsche Syntax: Phänomene und Entwicklungen". In: Dittli, Beat (ed.). Gömmer Migro? Freiburg(= Germanistica Friburgensia 18): 39-66.

Haas, Walter (1995): "Wenker contra Bremer oder: Empirie und Theorie des Dialekts". In: Cajot, Jose/Kremer, Ludger/Niebaum, Hermann (eds.): Lingua Theodisca. Beiträge zur Sprach- und Literaturwissenschaft. Jan Goosens zum 65. Geburtstag. Münster: 331-340.

Herrgen, Joachim (2001): "Dialektologie des Deutschen". In: Sylvain Auroux et al. (eds.) HSK. Geschichte der Sprachwissenschaften. Berlin/New York: 1513-1535.

Knoop, Ulrich (1982): "Die Marburger Schule: Entstehung und frühe Entwicklung der Dialektgeographie". In: Besch, Werner et al. (eds.): Dialektologie. Ein Handbuch zur deutschen und allgemeinen Dialektforschung. 1. Halbband. Berlin/New York: 38-92.

König, Werner (1978): DTV-Atlas zur deutschen Sprache. München.

Löffler, Heinrich (2003): Dialektologie. Eine Einführung. Tübingen.

Maurer, Friedrich (1926): Untersuchungen über die deutsche Verbstellung in ihrer geschichtlichen Entwicklung. Heidelberg.

Mitzka, Walter (1952): Handbuch zum Deutschen Sprachatlas. Marburg.

Mitzka, Walter/Martin, Bernhard (eds.) (1934): Deutscher Sprachatlas. Text zur 7. Lieferung. Marburg/Lahn.

Ruoff, Arno (1965): "Wenkersätze auf Tonband?" In: Sprachen - Zuordnung - Strukturen. Den Haag: 94-113.

Schirmunski, Viktor M. (1959): Nemeckaja dialektologija. Moskau/Leningrad.

Schwarz, Ernst (1950): Die deutschen Mundarten. Göttingen.

Seiler, Guido (im Druck): "Wie verlaufen syntaktische Isoglossen, und welche Konsequenzen sind daraus zu ziehen?" In: Eggers, Eckhardt/Schmidt, Jürgen Erich/Stellmacher, Dieter (eds.): Moderne Dialekte - neue Dialektologie. Stuttgart. 
Wenker, Georg (1881): Sprach-Atlas von Nord- und Mitteldeutschland. Einleitung. Strassburg/London.

Werlen, Iwar (1994): "Neuere Fragestellungen in der Erforschung der Syntax deutscher Dialekte". In: Mattheier, Klaus/Wiesinger, Peter (eds.). Dialektologie des Deutschen. Forschungsstand und Entwicklungstendenzen. Tübingen: 49-75.

Wrede, Ferdinand/Martin, Bernhard (eds.) (1932): Deutscher Sprachatlas. Text zur 6. Lieferung. Marburg/Lahn.

\section{Ungedruckte Quellen:}

Brief vom Eidgenössischen Departement des Innern an den Regierungsrat vom 06.05.1932 (SDS-Archiv).

Brief vom Eidgenössischen Departement des Innern an das Eidgenössische Politische Departement. Bern, 14.11.1932 (SDS-Archiv).

Brief vom Eidgenössischen Politischen Departement an die Deutsche Gesandschaft, Bern, 18.11.1932 (DSA-Archiv).

Brief von Bernhard Martin an Prof. Bachmann. Marburg, 18.03.1933 (DSA-Archiv).

\section{Online-Quellen:}

Schmidt, Jürgen E./Herrgen, Joachim (eds.) (2001-): Digitaler Wenker-Atlas (DiWA). Bearbeitet von Alfred Lameli, Alexandra Lenz, Jost Nickel und Roland Kehrein, KarlHeinz Müller, Stefan Rabanus. Marburg: Forschungsinstitut für deutsche Sprache "Deutscher Sprachatlas".

http://www.diwa.info. 


\section{Anhang}

\section{Die 40 Sätze Nord- und Mitteldeutschlands sowie der späteren Erhebung} Süddeutschlands (nach Mitzka 1952: 14)

1. Im Winter fliegen die trocknen Blätter in der Luft herum.

2. Es hört gleich auf zu schneien, dann wird das Wetter wieder besser.

3. Tu Kohlen in den Ofen, daß die Milch bald an zu kochen fängt.

4. Der gute alte Mann ist mit dem Pferde durchs Eis gebrochen und in das kalte Wasser gefallen.

5. Er ist vor vier oder sechs Wochen gestorben.

6. Das Feuer war zu stark, die Kuchen sind ja unten ganz schwarz gebrannt.

7. Er ißt die Eier immer ohne Salz und Pfeffer.

8. Die Füße tun mir sehr weh, ich glaube, ich habe sie durchgelaufen.

9. Ich bin bei der Frau gewesen und habe es ihr gesagt, und sie sagte, sie wollte es auch ihrer Tochter sagen.

10. Ich will es auch nicht mehr wieder tun!

11. Ich schlage dich gleich mit dem Kochlöffel um die Ohren, du Affe!

12. Wo gehst du hin? Sollen wir mit Dir gehen?

13. Es sind schlechte Zeiten.

14. Mein liebes Kind, bleib hier unten stehen, die bösen Gänse beißen dich tot.

15. Du hast heute am meisten gelernt und bist artig gewesen, du darfst früher nach Hause gehen als die andern.

16. Du bist noch nicht groß genug, um eine Flasche Wein auszutrinken, du mußt erst noch etwas wachsen und größer werden.

17. Geh, sei so gut und sag Deiner Schwester, sie sollte die Kleider für eure Mutter fertig nähen und mit der Bürste rein machen.

18. Hättest du ihn gekannt! dann wäre es anders gekommen, und es täte besser um ihn stehen.

19. Wer hat mir meinen Korb mit Fleisch gestohlen?

20. Er tat so, als hätten sie ihn zum Dreschen bestellt; sie haben es aber selbst getan.

21. Wem hat er die neue Geschichte erzählt?

22. Man muß laut schreien, sonst versteht er uns nicht.

23. Wir sind müde und haben Durst.

24. Als wir gestern Abend zurück kamen, da lagen die Andern schon zu Bett und waren fest am Schlafen.

25. Der Schnee ist diese Nacht bei uns liegen geblieben, aber heute Morgen ist er geschmolzen.

26. Hinter unserem Hause stehen drei schöne Apfelbäumchen mit rothen Äpfelchen.

27. Könnt ihr nicht noch ein Augenblickchen auf uns warten, dann gehen wir mit euch.

28. Ihr dürft nicht solche Kindereien treiben!

29. Unsere Berge sind nicht sehr hoch, die euren sind viel höher.

30. Wieviel Pfund Wurst und wieviel Brod wollt ihr haben?

31. Ich verstehe euch nicht, ihr müßt ein bißchen lauter sprechen. 
32. Habt ihr kein Stückchen weiße Seife für mich auf meinem Tische gefunden?

33. Sein Bruder will sich zwei schöne neue Häuser in eurem Garten bauen.

34. Das Wort kam ihm vom Herzen!

35. Das war recht von ihnen!

36. Was sitzen da für Vögelchen oben auf dem Mäuerchen?

37. Die Bauern hatten fünf Ochsen und neun Kühe und zwölf Schäfchen vor das Dorf gebracht, die wollten sie verkaufen.

38. Die Leute sind heute alle draußen auf dem Felde und mähen.

39. Geh nur, der braune Hund tut dir nichts.

40. Ich bin mit den Leuten da hinten über die Wiese ins Korn gefahren. 